\title{
Variability Modules for Java-like Languages
}

\author{
Ferruccio Damiani \\ University of Turin \\ Department of Computer Science \\ Turin, Italy \\ ferruccio.damiani@unito.it
}

\author{
Reiner Hähnle \\ Technical University of Darmstadt \\ Department of Computer Science \\ Darmstadt, Germany \\ reiner.haehnle@tu-darmstadt.de
}

\author{
Eduard Kamburjan \\ University of Oslo \\ Department of Informatics \\ Oslo, Norway \\ eduard@ifi.uio.no
}

\author{
Michael Lienhardt \\ ONERA \\ Palaiseau, France \\ michael.lienhardt@onera.fr
}

\author{
Luca Paolini \\ University of Turin \\ Department of Computer Science \\ Turin, Italy \\ luca.paolini@unito.it
}

\begin{abstract}
A Software Product Line (SPL) is a family of similar programs (called variants) generated from a common artifact base. A Multi SPL (MPL) is a set of interdependent SPLs (i.e., such that an SPL's variant can depend on variants from other SPLs). MPLs are challenging to model and implement efficiently, especially when different variants of the same SPL must coexist and interoperate. We address this challenge by introducing variability modules (VMs), a new language construct. A VM represents both a module and an SPL of standard (variabilityfree), possibly interdependent modules. Generating a variant of a VM triggers the generation of all variants required to fulfill its dependencies. Then, a set of interdependent VMs represents an MPL that can be compiled into a set of standard modules. We illustrate VMs by an example from an industrial modeling scenario, formalize them in a core calculus, provide an implementation for the Java-like modeling language ABS, and evaluate VMs by case studies.
\end{abstract}

\section{CCS CONCEPTS}

- Software and its engineering $\rightarrow$ Software product lines; Modules / packages; Classes and objects.

\section{KEYWORDS}

ABS language, Delta-oriented programming, Language design, Modules, Multi product line, Variant generation

\section{ACM Reference Format:}

Ferruccio Damiani, Reiner Hähnle, Eduard Kamburjan, Michael Lienhardt, and Luca Paolini. 2021. Variability Modules for Java-like Languages. In 25th ACM International Systems and Software Product Line Conference - Volume A (SPLC '21), September 6-11, 2021, Leicester, United Kingdom. ACM, New York, NY, USA, 12 pages. https://doi.org/10.1145/3461001.3471143

Permission to make digital or hard copies of all or part of this work for personal or classroom use is granted without fee provided that copies are not made or distributed for profit or commercial advantage and that copies bear this notice and the full citation on the first page. Copyrights for components of this work owned by others than ACM must be honored. Abstracting with credit is permitted. To copy otherwise, or republish, to post on servers or to redistribute to lists, requires prior specific permission and/or a fee. Request permissions from permissions@acm.org.

SPLC '21, September 6-11, 2021, Leicester, United Kingdom

(c) 2021 Association for Computing Machinery.

ACM ISBN 978-1-4503-8469-8/21/09 . \$ \$15.00

https://doi.org/10.1145/3461001.3471143

\section{INTRODUCTION}

Modeling variability aspects of complex software systems poses challenges currently not adequately met by standard approaches to software product line engineering (SPLE) [7, 30]. A first modeling challenge is the situation when more than one product line is involved and these product lines depend on each other. Such sets of related and interdependent product lines are known as a multi product line (MPL) [18, 44]. A second modeling challenge, orthogonal to MPLs, is the situation when different product variants from the same product line need to co-exist in the same context and must be interoperable [10].

In Sect. 2 we exemplify these two challenges in the context of an industrial case study from the literature [22, 24], performed for Deutsche Bahn Netz AG, where: (i) several interdependent product lines for networks, signals, switches, etc., occur; and (ii) for example, mechanic and electric rail switches are different variants of the same product line, and some train stations include both. Overall, MPLs give rise to the quest for mechanisms for hiding implementation details, reducing dependencies, controlling access to elements, etc. [18].

We take the standard concept of a module [45], used to structure large software systems since the 1970s, as a baseline. Software modules are supported in many programming and modeling languages, including ABS, Ada, Haskell, Java, Scala, to name just a few. Because modules are intended to facilitate interoperability and encapsulation, no further ad hoc concepts are needed for this purpose. We merely add variability to modules, rendering each module a product line of standard, variability-free modules. We call the resulting language concept variability module (VM).

The main advantage of VMs is their conceptual simplicity: as a straightforward extension of standard software modules, they are intuitive to use for anyone familiar with modules and with software product lines. Each VM is both a module and a product line of modules. This reduction of concepts not only drastically simplifies syntax, but reduces the cognitive burden on the modeler. We substantiate this claim: in Sect. 2 we illustrate the railways MPL case study in terms of VMs without the need to introduce any formal concepts. Nevertheless, there are a number of fundamental design decisions to take in the VM design. These are motivated and discussed in Sect. 3. 
We formulate the VM concept as an extension of the standard concept of module for Java-like (i.e., object-oriented, class-based and strongly typed) languages. To support variability, VMs employ delta-oriented programming (DOP) [1, Sect. 6.6.1], [32]. Specifically, we contribute (i) a theoretical foundation of VMs, including formal syntax and semantics, in terms of a core calculus; and (ii) an implementation of VMs as an extension of the ABS language [17, 21].

We choose ABS because it features native implementations of DOP and it was successfully used in industrial case studies for variability modeling $[24,28,46]$. We stress that VMs can be added on top of any Java-like language. For instance, a proof-of-concept realization of VM for the Java programming language, based on architectural patterns, is (informally) described in [39]. That paper demonstrates the usefulness of the VM concept, but lacks several VM features introduced here, as well as a formal foundation.

The formal underpinnings of VMs are covered in Sects. 4-7. In Sect. 4 we declare the VM syntax and spell out consistency requirements. Sect. 5 formalizes a statically checkable property of VMs: the principle of encapsulated variability, which ensures that any dependency among VMs can be reduced to dependencies among standard variability-free modules. In Sect. 6 we define variant generation in terms of a "flattening" semantics: the variants requested from an SPL represented by a VM, together with necessary variants of other VMs it depends upon, are generated by translating each VM into a set of variability-free, standard modules (one per variant). This results in a variability-free program with suitably disambiguated identifiers and is sufficient to define the semantics of VMs precisely, to compile and to run them. In Sect. 7 we prove soundness of flattening.

Sect. 8 describes how the VM concept is integrated into the existing ABS tool chain. As long as one has control over the parser and abstract syntax tree, it is relatively straightforward to realize the flattening algorithm of Sect. 6 within any compiler tool chain. We evaluate VMs by means of case studies. Related work is discussed in Sect. 9, we conclude in Sect. 10 by outlining ongoing work.

\section{INTRODUCING VARIABILITY MODULES}

We illustrate VMs with an example based on an industrial case study from railway engineering [24].

Our scenario contains signals, switches, interlocking systems, that use multiple variants of signals and switches, and a railway station that uses multiple variants of interlocking systems. Fig. 1 shows the

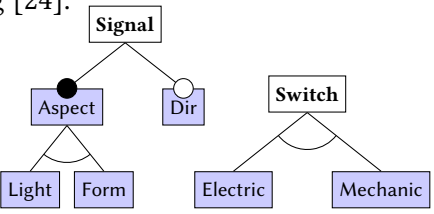

Figure 1: Features of Signals and Switches. feature models for switches and signals. ${ }^{1}$ A signal is either a light signal, using bulbs and colors to indicate the signal aspect or a form signal that uses mechanically moved shapes. All variants of signal have the interface to the interlocking system and basic functionality, such as aspect change, in common (e.g., signals have always the

\footnotetext{
${ }^{1}$ Feature models [8] specify software variants in terms of features. A feature is a name representing some functionality, a set of features is called a configuration, and each variant is identified by a valid configuration (called a product, for short). Equivalent representations of feature models have been proposed in the literature $[1,2]$, like feature diagrams (Fig. 1) and propositional formulas (lines with keyword features in Figs. 2-3).
}

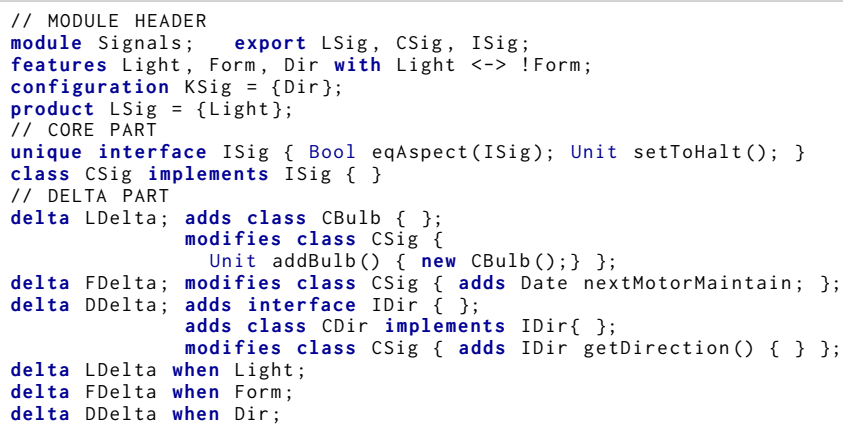

Figure 2: An SPL of signals as a VM.

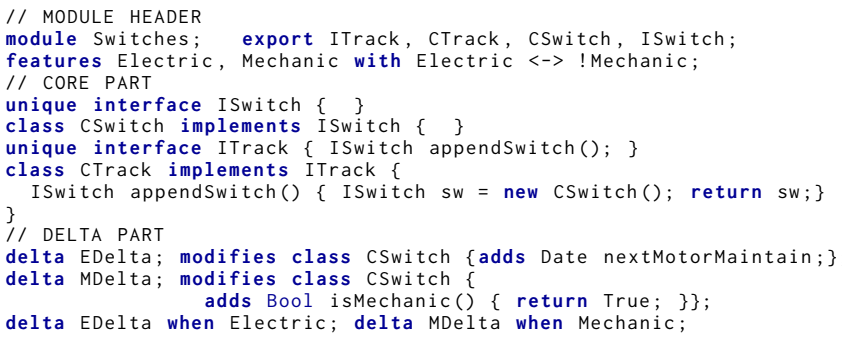

Figure 3: An SPL of tracks and switches as a VM.

aspects "Halt" and "Go"). If multiple outgoing tracks are possible, a signal may also indicate the direction the train is going-so there are 4 signal variants. Variability shows, for example, in the presence of an additional class Bulb in the light signals variant and in the fact that method setToHalt (which changes the shown aspect to "Halt") is different for form signals and light signals (the latter communicate with their Bulb instances).

Signals are modeled by the VM signals in Fig. 2. It starts with the module header, comprising: the keyword module; the module name; the list of exported module elements; the feature list constrained with a propositional formula describing the products; a list of configuration definitions (here just one); and a list of product definitions (here just one), where each definition gives a name to a set of features. Next is the module core part, comprising declarations of interface Isig and of class csig that implements Isig. By default, class and interface definitions can be modified/removed by deltas to obtain different product variants, however, class/interface definitions annotated by the keyword unique must be the same in all product variants. They enable interoperability between different product variants of the same VM. Finally there is the delta part, comprising the deltas that describe the implementation of different variants and their application conditions. Classes and interfaces added by deltas can be modified or removed again by other deltas. The delta LDelta, triggered by feature Light, adds a class Bulb and modifies the class csig to reference the class Bulb. Deltas FDelta and DDelta implement features Form and Dir, respectively.

Switches and tracks are modeled by the VM in Fig. 3. It is structurally similar to signals. A switch is either electric (controlled from 


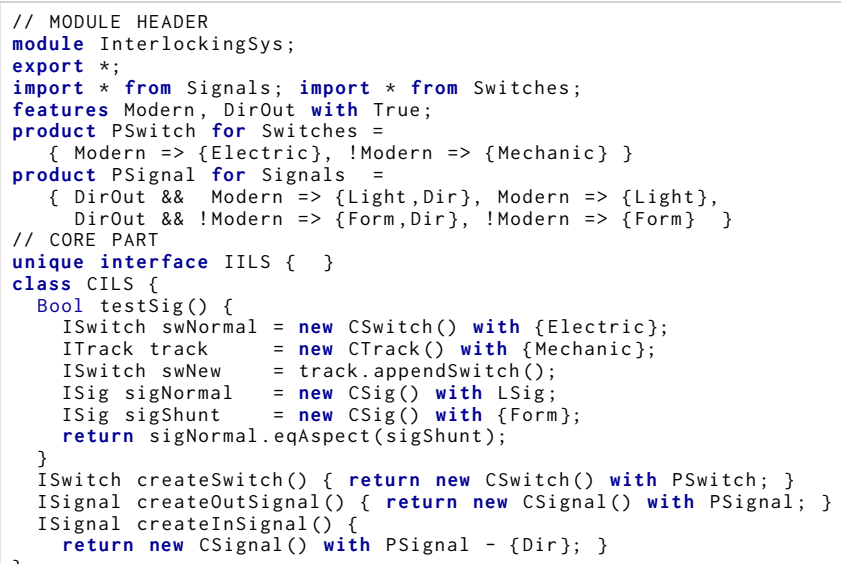

Figure 4: An SPL of interlocking systems as a VM.

the interlocking system) or mechanic (controlled locally by a lever). Class ctrack contains a reference to class cswitch, which is not declared unique. So, even though class cTrack is not modified/removed by any delta, its declaration cannot be annotated with unique.

Interlocking systems are modeled by the VM in Fig. 4. An interlocking system manages switches and signals that lie on tracks, it imports all the exported elements (feature names are implicitly exported/imported) of the VMs signals and switches. The VM Interlockingsys has four variants, modeled by two optional features. Line 6 contains a product definition that gives name Pswitch to a product of the VM switch. It is called an open product definition, because it depends on the selected product of the VM Interlockingsys itself: if feature Modern is selected PSwitch specifies an electric switch, otherwise it specifies a mechanic switch (product clauses are evaluated in order until a valid one is found). Line 8 contains an open product definition for the VM signal. It is worth observing that open product definitions enable implementing different variants of the VM Interlockingsys even without using deltas. Method testsig of class cILS instantiates classes from two different product variants of switches and from two different product variants of signals. All references to non-unique imported classes/interfaces specify a product, by using a with clause. In a with clause, the product can be specified by explicitly listing its features, by using one of the defined product names, or (more generally) by a set-theoretic expression. For example, track is taken from product \{Mechanic\} of module switches, while signormal uses the product name Lsig imported from signals. In line 17 a switch is added to a track element: since track contains a reference to an instance of the mechanic variant of class CTrack, appendSwitch() will add a mechanic switch. All signal variants of the VM signals share the same definition of the unique Isig interface, thus making it accessible to anyone that imports it from signals. On the other hand, the cBulb class is only used inside the VM signals. Different product variants are fully interoperable, as witnessed by the expression in line 20.

A VM that does not contain a feature model (and, therefore, no configuration definitions, no open product definitions and no deltas) is called a variability-free module (VFM). All classes of a

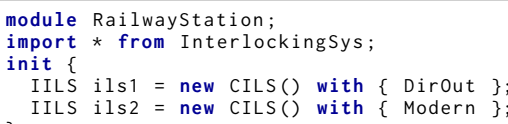

Figure 5: Railway station as a main module.

VFM are considered unique (there is no need to write the unique keyword). Each program must have exactly one main module: a VFM containing an implicit class providing an initialization method declared by the keyword init. The whole railway station is modeled by a main module, given in Fig. 5, together with the VM signals in Fig. 2, the VM switches in Fig. 3, and the VM Interlockingsys in Fig. 4. It represents a MPL-we call it the railway station MPL.

\section{DESIGN DECISIONS}

Below we briefly illustrate the rationale behind the major VM design decisions.

Unique Annotation. As explained above, unique class/interface declarations in a VM $\mathrm{m}$ are shared by all variants of $\mathrm{m}$. Without the unique keyword, unique class/interface declarations should be inferred (cf. Def. 7.1, Thm. 7.1 below), thus creating the danger of unintended changes of the set of unique classes/interfaces in a program. Obviously, a tool that points out all class/interface declarations that could be annotated unique would be useful.

Principle of Encapsulated Variability (PEV). The PEV prescribes that each VM can depend on other VMs only by using classes or interfaces that are either unique or that belong to a specific variant. If a VM program adheres to the PEV, then flattening (defined in Sect. 6) - which removes variability and generates those variants required by the dependencies-can resolve all dependencies among VMs to dependencies among VFM. The main reasons for adopting the PEV are simplicity and usability: it suffices to work with a standard module concept (no need for composition or disambiguation operators as, for example, [26]) and it is easy for the modeler to find out to which implementation any object reference in a VM refers to.

Local Feature Model. Each VM has its own feature model disjoint from those of other VMs: each feature name is local to the VM where it is declared, and there is no global name space for features. It might be useful to add support for expressing constraints connecting the local feature models (e.g., to specify that certain variants must not co-exist in the same application).

Implicit Export/Import Flattening. Each VM $\mathrm{m}$ must declare the union of the exports/imports of all its variants. Then the flattening generates the export/import clauses of each variant by dropping export clauses for classes/interfaces not present in that variant, and by creating the import clauses for the required variants of the VMs mentioned in the import clauses of $\mathrm{m}$. This design choice avoids the need to define delta operations on export/import clauses in the language that then would be supplied by the modeler. ${ }^{2}$ Altogether,

\footnotetext{
${ }^{2}$ To extend VMs with delta operations on export clauses is straightforward. It would allow sometimes to shorten export clauses. On the other hand, since implicit flattening drops all unused imports, deltas on import clauses provide no advantage.
} 


\begin{tabular}{|c|c|c|}
\hline $\begin{array}{l}\text { Prg } \\
\text { Mdl }\end{array}$ & $\begin{array}{l}::=\overline{\operatorname{Mdl}} \\
::=\operatorname{MdlH} \operatorname{MdlC} \operatorname{MdlD}\end{array}$ & $\begin{array}{l}\text { Program } \\
\text { (Variability) Module }\end{array}$ \\
\hline \multicolumn{3}{|c|}{ MdlH $::=$ module $M ;[$ export $\mathrm{tc} ;]$} \\
\hline & $\overline{\text { import tC from } M ;}[$ features $\bar{F}$ with $\Phi ; \overline{\mathrm{KD}}] \overline{\mathrm{PD}}$ & Module Header \\
\hline $\mathrm{tc}$ & $::=\mathrm{tN} \overline{\mathrm{tN}} \mid *$ & Trade Clause \\
\hline $\mathrm{tN}$ & $::=c|\mathrm{I}| \mathrm{K} \mid \mathrm{P}$ & Traded names \\
\hline KD & $::=$ configuration $\mathrm{K}=\mathrm{KE}$ & Configuration Declaration \\
\hline KE & $::=\mathrm{K}|\mathrm{P}|\{\overline{\mathrm{F}}\}|\mathrm{KE}+\mathrm{KE}| \mathrm{KE} * \mathrm{KE} \mid \mathrm{KE}-\mathrm{KE}$ & Configuration Expression \\
\hline PD & $::=$ product $P[$ for $M]=K E$ & Closed Product Declaration \\
\hline & $\mid$ product $P[$ for $M]=\{\mathrm{PC}, \overline{\mathrm{PC}}\}$ & Open Product Declaration \\
\hline $\mathrm{PC}$ & $::=\Phi \Rightarrow \mathrm{KE}$ & Pattern Clause \\
\hline \multicolumn{2}{|c|}{$\operatorname{MdlC}::=\overline{\operatorname{Defn}}[$ init $\{\overline{\mathrm{s}}\}]$} & Module Core Part \\
\hline Defn & $::=[$ unique $]$ ID $\mid[$ unique $] C D$ & Interface/Class Definition \\
\hline ID & $::=$ interface $\mathrm{I}[$ extends IR $\overline{\mathrm{IR}}]\{\overline{\mathrm{MH}}\}$ & Interface Definition \\
\hline IR & $::=\mathrm{I}[$ with $\mathrm{KE}] \mid$ M.I $[$ with KE $]$ & Interface Reference \\
\hline MH & $::=\mathrm{T} \mathrm{m}(\overline{\mathrm{T} x})$ & Method Header \\
\hline $\mathrm{T}$ & \begin{tabular}{l|l|l|l}
$::=$ IR & Unit $\mid$ Int $\mid \ldots$ \\
\end{tabular} & Type \\
\hline$C D$ & $::=$ class $C \quad[$ implements $\mathrm{IR} \overline{\mathrm{IR}}]\{\overline{\mathrm{FD}} \overline{\mathrm{MD}}\}$ & Class Definition \\
\hline FD & $::=\mathrm{T} f$ & Field Definition \\
\hline MD & $::=$ MH $\{\overline{\mathrm{S}}$ return $\mathrm{E} ;\}$ & Method Definition \\
\hline s & $::=[\mathrm{T}] \mathrm{v}=\mathrm{E} ;|\mathrm{E} . \mathrm{f}=\mathrm{E} ;| \ldots$ & Statement \\
\hline E & $::=x \mid$ E.f $|E \cdot m(\bar{E})|$ new $\operatorname{CR}()[$ with KE $] \mid \ldots$ & Expression \\
\hline $\mathrm{CR}$ & $::=\mathrm{c} \mid$ M.C & Class Reference \\
\hline MdlD & $::=\overline{\mathrm{Dlt}} \mathrm{CK}$ & Module Delta Part \\
\hline $\mathrm{Dlt}$ & $::=\operatorname{delta} \mathrm{D} ; \overline{\mathrm{CO}} \overline{\mathrm{IO}}$ & Delta \\
\hline co & $\begin{array}{l}::=\text { adds } C D \mid \text { removes class } C \\
\mid \text { modifies class } C[\text { adds IR } \overline{I R}][\text { removes IR } \overline{I R}]\{\overline{A 0}\}\end{array}$ & Class Operation \\
\hline AO & $::=$ adds $A D \mid$ removes $H D \mid$ modifies MD & Attribute Operation \\
\hline AD & $::=\mathrm{FD} \mid \mathrm{MD}$ & Attribute Declaration \\
\hline HD & $::=\mathrm{FD} \mid \mathrm{MH}$ & Header Declaration \\
\hline I0 & $\begin{array}{l}::=\text { adds ID } \mid \text { removes interface } I \\
\mid \text { modifies interface } I[\text { adds IR } \overline{I R}][\text { removes IR } \overline{\mathrm{IR}}]\{\overline{\mathrm{SO}}\}\end{array}$ & Interface Operation \\
\hline so & $::=$ adds $M H \mid$ removes $M H$ & Signature Operation \\
\hline CK & $::=\overline{\mathrm{DAC}} \overline{\mathrm{DAO}}$ & Configuration Knowledge \\
\hline DAC & $::=$ delta $\mathrm{D}$ when $\Phi$ & Delta Activation Condition \\
\hline DAO & $::=\mathrm{D} \overline{\mathrm{D}}<\mathrm{D} \overline{\mathrm{D}} \overline{<\mathrm{D} \overline{\mathrm{D}}}$ & Delta Application Order \\
\hline
\end{tabular}

Figure 6: ABS-VM abridged syntax.

it reduces the cognitive burden to understand VM code and the effort to write it.

Family-based checking. VM are designed to permit familybased analysis [15, 42, 43]. The implementation of VM as part of the ABS compiler tool chain (Sect. 8) checks PEV before flattening. Moreover, we are currently implementing a family-based analysis to check-before flattening-whether a program Prg is delta-application sound (thus ensuring, according to Thm. 7.4, that flattening will succeed), whether the generated VFM will be well-typed, and, more generally, whether the variants of the VMs in Prg as a whole (including those not generated by flattening Prg) would form a well-typed VFM.

\section{SYNTAX OF ABS VARIABILITY MODULES}

The abridged syntax of $A B S$ with VMs (ABS-VM, for short) is given in Fig. 6. It defines the $O O$ fragment of $A B S$ [17, 21], extended with VM concepts. A program is a sequence of VMs-as usual, $\bar{X}$ denotes a possibly empty finite sequence of elements $X$. A VM consists of a header (Md1H), a core part (Md1C), and a delta part (Md1D). A VM header comprises the keyword module followed by the name of the
VM, by some (possibly none) import and export clauses (listing the class/interface/configuration/product names, respectively, that are exported or imported by the VM), by the optional definition of a feature model (where $\bar{F}$ are the features and $\Phi$ is a propositional formula over features), by a list of configuration definitions and by a list of product definitions. A configuration expression KE is a settheoretic expression over sets of features $(+, *$ and - denote union, intersection and difference, respectively). A product definition PD is closed if it is of the form product $\mathrm{P}[$ for $\mathrm{M}]=\mathrm{KE}$, otherwise it is open. The clauses in an open product definition are examined in sequence until the first valid clause is found. The right-hand sides of configuration definitions do not contain product names, and the right-hand sides of closed product definitions do not contain open product names. Recursive configuration/product definitions are forbidden.

Both the module core part and the module delta part may be empty. A module core part comprises a sequence of class and interface definitions $\overline{\text { Defn. }}$ As an extension to ABS syntax, each of these definitions may be prefixed by the keyword unique. Each use of a class, interface or product name imported from another module may be prefixed by the name of the module-the name of the module must be used if there are ambiguities (for example, when an interface with name I is imported from two different modules). Moreover, each use of a non-unique class or interface imported from another module must be followed by a with-clause, specifying (by means of a configuration expression) the variant of the VM it is taken from. From now on, we consider only ABS-VM programs containing one main module (as in the final paragraph of Sect. 2) such that any other VM does not contain the keyword init. Observe that a VFM (as defined in the final paragraph of Sect. 2) without a product definition (a VFM may contain closed product definitions) and no occurrences of the with keyword is a variability-free $A B S$ module.

The module delta part comprises a sequence of delta definitions $\overline{\mathrm{Dlt}}$ followed by configuration knowledge cк. Each delta specifies a number of changes to the module core part. A delta comprises the keyword delta followed by the name of the delta, by a sequence of class operations $\overline{c 0}$ and by a sequence of interface operations $\overline{\mathrm{I} o}$. An interface operation can add or remove an interface definition, or modify it by adding/removing names to the list of the extended interfaces or by adding/removing method headers. A class operation can add or remove a class definition, or modify it by adding/removing names to the list of the implemented interfaces, by adding/removing fields or by adding/removing/modifying methods. Modifying a method means to replace its body with a new body. The new body may call the reserved method name original, which during delta application is bound to the previous implementation of the method.

Configuration knowledge ск provides a mapping from products to variants by describing the connection between deltas and features: it specifies an activation condition $\Phi$ (a propositional formula over features) for each delta $\mathrm{D}$ by means of a DAC clause; and it specifies an application ordering between deltas by means of a sequence of DAO clauses. Each DAO clause specifies a partial order over the set of deltas in terms of a total order on disjoint subsets of delta names-a DAO clause allows developers to express (as a partial order) dependencies between the deltas (which are usually semantic "requires" relations [4]). The overall delta application order is the union of these partial orders-the compiler checks that the resulting relation 
$R$ represents a specification that is consistent (i.e., $R$ is a partial order) and unambiguous (i.e., all the total delta application orders that respect $R$ generate the same variant for each product). Techniques for checking that $R$ is unambiguous are described in the literature [4, 27].

The following definition of normal form formalizes a minimum consistency requirement on ABS-VM programs.

Definition 4.1 (ABS-VM Normal Form). An ABS-VM program Prg is in normal form if all its VMs $\mathrm{M}$ satisfy the following conditions:

(1) All class references CR and interface references IR occurring in $M$ are qualified, that is of the form $M^{\prime} . N$ for some module name $M^{\prime}$ and class/interface name $N$, and if $M^{\prime} \neq M$ then $M$ contains the import clause import $\mathrm{N}$ from $\mathrm{M}^{\prime}$.

(2) If $\mathrm{M}$ contains a clause import tc from $\mathrm{M}^{\prime}$ then $\mathrm{M}^{\prime} \neq \mathrm{M}$ and all traded names in tc occur in the export clause of $\mathrm{M}^{\prime}$. No open product names are exported.

(3) Let $\mathrm{m}$ contain a definition

(a) configuration $\mathrm{K}=\mathrm{KE}$. Then: (i) all feature names occurring in KE are features of $M$ and all configuration names occurring in KE have been already defined in $\mathrm{M}$; and (ii) no product name occurs in KE.

(b) product $\mathrm{P}=\mathrm{KE}$ then: (i) condition (3a).(i) above holds; (ii) all product names occurring in KE have been already defined in $M$ and are closed; and (iii) $K E$ denotes a product of $M$.

(c) product $P$ for $M^{\prime}=K E$. Then: (i) $M \neq M^{\prime}$; (ii) all feature names occurring in KE are features of $M^{\prime}$ and all configuration/product names occurring in KE are imported from $\mathrm{M}^{\prime}$; and (iii) KE denotes a product of $M^{\prime}$.

(d) product $P$ for $M^{\prime}=\left\{\Phi_{1} \Rightarrow K_{1}, \ldots, \Phi_{n} \Rightarrow K_{n}\right\}(n \geq 1)$. Then: (i) $M \neq$ $M^{\prime}$; (ii) for all $1 \leq j \leq n$, all configuration/product names occurring in $\mathrm{KE}_{j}$ are imported from $\mathrm{M}^{\prime}$; (iii) for all $1 \leq j \leq n$, all feature names occurring in $\Phi_{j}$ are features of $\mathrm{M}$, all feature names occurring in $\mathrm{KE}_{j}$ are features of $\mathrm{M}^{\prime}$, at least one product of $\mathrm{M}$ satisfies $\left(\bigwedge_{1 \leq i<j} \neg \Phi_{i}\right) \wedge \Phi_{j}$, and $\mathrm{KE}_{j}$ denotes a product of $\mathrm{M}^{\prime}$; and (iv) all products of $\mathrm{M}$ satisfy $\bigvee_{1 \leq i \leq n} \Phi_{i}$.

(e) product $\mathrm{P}=\left\{\Phi_{1} \Rightarrow>\mathrm{KE}_{1}, \ldots, \Phi_{n} \Rightarrow \mathrm{KE}_{n}\right\}(n \geq 1)$. Then: (i) for all $1 \leq j \leq n$, all configuration/product names occurring in $\mathrm{KE}_{j}$ have been already defined in $\mathrm{M}$; (ii) the condition obtained from condition (3d).(iii) above by replacing $\mathrm{M}^{\prime}$ by $M$ holds; and (iii) condition (3d).(iv) above holds.

(4) If $M$ contains an occurrence of new $M^{\prime} . C\left(\right.$ ) with KE Or $M^{\prime}$. I with KE, then: (i) all feature names occurring in KE are features of $M^{\prime}$; (ii) if $M^{\prime}=M$ then all configuration/product names occurring in KE are defined in $M$; (iii) if $M^{\prime} \neq M$ then all configuration/product names occurring in KE are either imported from $M^{\prime}$ or defined in $M$ by a declaration of the form product $P$ for $\mathrm{M}^{\prime}=\ldots$; and (iv) $K E$ denotes a product of $M^{\prime}$.

Checking whether a program can be transformed into normal form (and, if so, doing it) is straightforward-for each VM M: conditions (1), (3a), (3b), (3e) and (when $M^{\prime}=m$ ) condition (4) can be ensured by inspecting only $\mathrm{M}$ condition (2) and (when $\mathrm{M}^{\prime} \neq \mathrm{M}$ ) condition (4) can be ensured by inspecting only $м$ and the header of the modules $M^{\prime}$ mentioned in the import clause of $\mathrm{M}$. Conditions (3b).(iii), (3c).(iii), (3d).(iii), (3d).(iv) and (4).(iv) can be checked with a SAT solver [41] Programs that cannot be transformed into normal form are rejected by the compiler.

\section{ENCAPSULATED VARIABILITY}

In this section we formalize the principle of encapsulated variability. We only consider programs in normal form (Def. 4.1). To formalize and to implement automated checking of PEV adherence we introduce the notion of dependency and the functions CORE, UNIQUE, and BASE.

Definition 5.1 (Dependency). A VM ${ }^{\prime}$ depends on a VM $\mathrm{M}$ if $\mathrm{M}^{\prime}$ contains an occurrence of M.N where $\mathrm{N}$ is a class/interface name. An occurrence of M.I with KE or new M.C() with KE is called with-dependency (on M.I or M.c, respectively), while an occurrence of M.I or new M.C(...) (i.e. not followed by a with) is called with-free-dependency (on M.I or M.C, respectively). An occurrence of M.I with KE Or M.C() with KE is called with-open-dependency if KE contains an occurrence of an open product name $\mathrm{P}$, it is called with-closed-dependency else.

Definition 5.2 (Functions CORE, UNIQUE, BASE). Given a program Prg; for all VMs $M$ of Prg: CORE(Prg, M) is the set of qualified names M.N of all interfaces/classes $N$ whose definition occurs in the core part of $M$; UNIQUE( $\operatorname{Prg}, M) \subseteq \operatorname{CORE}(\operatorname{Prg}, M)$ contains those class/interface names whose declaration is annotated with unique; $\operatorname{BASE}(\operatorname{Prg}, M) \subseteq \operatorname{CORE}(\operatorname{Prg}, M)$ contains those class/interface names that are modified, removed or added by some delta of $M$.

Now we can formalize the PEV as follows:

Definition 5.3 (Principle of Encapsulated Variability (PEV)). A program Prg (in normal form) adheres to PEV, if for all VMs $M$ of Prg:

(1) UNIQUE $(\operatorname{Prg}, M) \cap \operatorname{BASE}(\operatorname{Prg}, M)=\emptyset$.

(2) For all M.N $\in$ UNIQUE(Prg, M) the definition Defn of $N$ (in the core part Mdic of $M$ ) does not contain with-open-dependencies and, for all with-free-dependencies on M.N' occurring in Defn, it holds that M.N' $\in$ UNIQUE(Prg, M).

(3) For all with-free-dependencies on $M^{\prime} . N$ occurring in $M$ : if $M^{\prime} \neq M$ then $M^{\prime} . N \in$ UNIQUE $\left(\operatorname{Prg}, M^{\prime}\right)$.

To check whether a program adheres to the PEV is straightforward. Programs that do not adhere to the PEV are rejected by the compiler. According to the PEV, VMs can support two types of interaction among variants:

Variant interoperability. Different variants of the same VM can coexist and cooperate via unique classes/interfaces. For instance, in the interlocking system MPL of Sect. 2, all interfaces are unique and all classes are not unique (which is a common pattern). Then, in line 20 of Fig. 4, an instance of class csig in the variant of VM Signal for product $\{$ Light $\}$ receives an invocation of method eqAspect that (accepts an argument of type signal as formal parameter and) takes as parameter an instance of csig in the variant of signal for product $\{$ Form\}.

Variant interdependence. The code of a variant of a $\mathrm{VM} \mathrm{M}_{1}$ can depend on the code of a variant of a $\mathrm{VM} \mathrm{M}_{2}$ (and possibly vice versa). I.e., the code of $M_{1}$ refers to unique classes/interfaces of $M_{2}$ (via with-free-dependencies) or to classes/interfaces of a specific variant of $M_{2}$ (via with-dependencies). A special case of variant interdependence is when $M_{1}=M_{2}$, i.e., $M_{1}$ has a with-dependency on a class/interface of $M_{1}$ itself. Then in the flattened program a variant of $M_{1}$ will contain an occurrence of a class/interface name that is declared in a different variant of $M_{1}$. 


\section{SEMANTICS OF VARIABILITY MODULES}

In this section, we assume (without loss of generality): (i) the considered program Prg is in normal form and adheres to the PEV; (ii) all configuration definitions КD and closed product definitions PD have been resolved in Prg, i.e. all occurrences of their names are removed from export/import clauses of Prg, and all their remaining occurrences in Prg were replaced with their value (a set of features).

In Sect. 6.1 we introduce auxiliary functions for the extraction of relevant information from ABS-VM programs. Then, in Sect. 6.2 we give rewrite rules for transforming an ABS-VM program into a variability-free $A B S$ program.

\subsection{Auxiliary Functions}

Definition 6.1 (Lookup Functions). Given a VM м of Prg we define the sets:

- mdlUnique(Prg, $\mathrm{M})$ for the interface/class definitions $\overline{\text { Defn }}$ in the Module Core Part mdic of $\mathrm{m}$ annotated with unique.

- mdINotUnique(Prg, M) for the interface/class definitions not in mdlUnique( $\operatorname{Prg}, M)$.

- mdllnit(Prg, M) for the init block of $M$, if it exists, or the empty sequence otherwise; also mdllnit(Prg) for the name $M$ of the VM such that mdllnit(Prg, $M$ ) is not the empty sequence.

- $\operatorname{mdIDelta}(\operatorname{Prg}, \mathrm{M}, \pi)$ where $\pi$ is a product of $\mathrm{M}$, for any ordered sequence $\overline{\text { DIt }}$ containing exactly those deltas of $m$ that are activated by $\pi$, respecting the order among deltas specified in the configuration knowledge of $\mathrm{M}$.

The meaning of with-free-dependencies or open with-dependencies $\delta$ occurring in a VM $\mathrm{M}$ is relative to the product $\pi$ of $\mathrm{m}$ being considered. We say a dependency $\delta$ is ground to mean that it is either with-free or the configuration expression KE in its with-clause is a set of features $\pi=\left\{F_{1}, \ldots, F_{n}\right\}(n \geq 0)$. The following definition introduces notations for extracting the meaning of ground dependencies occurring in the core part of a given VM of a given program.

Definition 6.2 (Ground Dependency Meaning). Given a program Prg, a VM $\mathrm{M}$ of Prg, a ground dependency $\delta$ on $\mathrm{M}^{\prime}$. N occurring in $\mathrm{M}$, let $x c$ be either the symbol $\perp$ or a product $\pi$ of . We define:

$$
\begin{aligned}
& \Downarrow\left(\operatorname{Prg}, M^{\prime}, \delta, x c\right)= \\
& \begin{cases}\left(M^{\prime}, \perp\right) & \text { when } M^{\prime} . N \in \text { UNIQUE }\left(\operatorname{Prg}, M^{\prime}\right) \\
\left(M^{\prime}, x c\right) & \text { when } M^{\prime}=M^{\prime} \delta \text { is with-free and } M^{\prime} . N \notin U N I Q U E\left(\operatorname{Prg}, M^{\prime}\right) \\
\left(M^{\prime}, \pi\right) & \text { when } \delta \text { is } M^{\prime} . N \text { with } \pi \text { and } M^{\prime} . N \notin U N I Q U E\left(\operatorname{Prg}, M^{\prime}\right)\end{cases}
\end{aligned}
$$

Let all the dependencies occurring in the core part Mdic of $\mathrm{M}$ be ground. Then $\Downarrow(\operatorname{Prg}, M, M d l c, x c)=\{\Downarrow(\operatorname{Prg}, M, \delta, x c) \mid \delta$ is a dependency in Mdlc $\}$.

Flattening a program Prg may require to generate more than one variant for each of its VMs. The flattening process generates new names for the generated variability-free ABS modules implementing the required variants, and translates the dependencies occurring in Prg to suitable dependencies using the generated names. The following definition introduces notations for the names of the generated modules and the translation of with- and with-free-dependencies into the corresponding dependencies among non-variable ABS modules.

Definition 6.3 (New Module Name, Dependency Translation). Given a program Prg, a VM $\mathrm{M}$ of Prg, let $x c$ be either $\perp$ or a product $\pi$ of $\mathrm{M}$. We denote by $\Uparrow(M, x c)$ the name of the module that implements the unique part of the variants of $\mathrm{m}$ if $x c=\perp$, or the name of the module that implements the non-unique part of the variant of $\mathrm{m}$ for product $\pi$. Moreover, given a ground dependency $\delta$ on $\mathrm{M}^{\prime} . \mathrm{N}$, we define:

$$
\begin{aligned}
& \Uparrow(\operatorname{Prg}, M, \delta, x c)= \\
& \left\{\begin{array}{l}
\pi\left(M^{\prime}, \perp\right) \cdot N \text { when } M^{\prime} . N \in \text { UNIQUE }\left(\operatorname{Prg}, M^{\prime}\right) \\
\pi\left(M^{\prime}, x c\right) \cdot N \text { when } M^{\prime}=M, \delta \text { is with-free and } M^{\prime} . N \notin U N I Q U E(\operatorname{Prg}, M) \\
\pi\left(M^{\prime}, \pi\right) \cdot N \text { when } \delta \text { is } M^{\prime} . N \text { with } \pi \text { and } M^{\prime} . N \notin U N I Q U E\left(\operatorname{Prg}, M^{\prime}\right)
\end{array}\right.
\end{aligned}
$$

Let all the dependencies occurring in the core part Mdic of $M$ be ground. Then we define $\Uparrow(\mathrm{Prg}, \mathrm{M}, \mathrm{Mdlc}, x c)$ as the VM core Mdlc' obtained from Mdlc by replacing each dependency $\delta$ occurring in it with $\Uparrow(\mathrm{Prg}, \mathrm{M}, \delta, x c)$.

\subsection{Flattening}

The following definition formalizes the application of an ordered sequence of deltas $\overline{\text { DIt }}$ (the deltas activated by a product $\pi$ of a VM м) to a sequence $\overline{\overline{D e f n}}$ of interface/class definitions (the non-unique class/interface definitions in the module core part Md1c of $\mathrm{M}$ ).

Definition 6.4 (Delta Application). Given a sequence of declarations $\overline{\text { Defn }}$ and an ordered sequence of deltas $\overline{\text { DIt }}$, we denote with the relation $(\overline{\text { Dlt }}, \overline{\text { Defn }}) \rightarrow^{*} \overline{\text { Defn' }^{\prime}}$ that $\overline{\text { Defn' }^{\prime}}$ is the outcome of the procedure described in App. A.

For all VMs $\mathrm{M}$ of Prg, if the products $\pi$ of $\mathrm{M}$ are given, then the right-hand side of each open product definition product $P=\cdots$ or product $P$ for $M^{\prime}=\cdots$ in $M$ can be evaluated to a product. This induces a mapping $\sigma=\operatorname{gen} \mathrm{P}(\operatorname{Prg}, \mathrm{M}, \pi)$ from open product names to products. Given a sequence of interface/class definitions $\overline{\text { Defn }}$ and such a mapping $\sigma$, for at least the open product names occurring in $\overline{\text { Defn }}$ to products, denote with $\sigma(\overline{\text { Defn }})$ the definitions obtained from $\overline{\text { Defn }}$ by replacing each occurrence of an open product name P with $\sigma(\mathrm{P})$.

Definition 6.5 (Grounding with-clauses). Given a Module Core Part Mdic that does not contain occurrences of product names, we write MdIc $\sim{ }^{*}$ MdIC' to mean that the module core part MdIc' has been obtained from Mdlc by replacing the right-hand side of each withclause (which is a set-theoretic expression over sets of features) by the corresponding set of features (which, since the overall program is assumed in normal-form, is a product).

We are ready to define the rules that flatten a VM:

Definition 6.6 (Flattening a VM). Let $\mathrm{m}$ be the name of a VM of Prg, let $x c$ be either the symbol $\perp$ or a product $\pi$ of $\mathrm{m}$. The following rules define a judgment of the form $M \stackrel{\operatorname{Prg}, x c}{\longrightarrow} D$, Mdl where: $\operatorname{Mdl}$ is the code of a variability-free ABS module named $\Uparrow(m, x c)$, which, for the case $x c=\perp$ implements the unique part of the variants of $\mathrm{M}$, for the case $x c=\pi$ implements the non-unique part of the variant of $\mathrm{M}$ for product $\pi$. Moreover, $D$ is a set that identifies the variability-free $\mathrm{ABS}$ modules that $\Uparrow(\mathrm{m}, x c)$ depends upon.

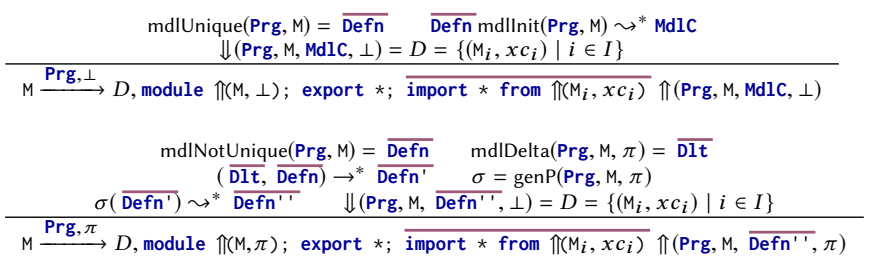


The first rule generates a module implementing the unique part of the variants of a given VM м. To do so, it extracts the unique part $\overline{\text { Defn }}$ of the VM, its optional init block, and the dependencies $D$ occurring in these parts. The rule returns the set of dependencies $D$ (which identifies variability-free ABS modules that need to be generated) and a new variability-free ABS module named $\pi(M, \perp)$ that: (i) exports everything; (ii) imports from all variability-free ABS modules identified in $D$; and (iii) contains the unique classes/interfaces of the original VM, where all syntactic dependencies have been translated according to $\Uparrow$.

The second rule generates a variability-free ABS module implementing the non-unique part of the variant of the VM $\mathrm{v}$ for the product $\pi$. It is similar to the first rule, except for two elements: (i) the optional init block is not considered (it cannot be present); (ii) the extracted (non-unique classes/interfaces) part of the VM is modified by applying the activated deltas as described in Def. 6.4 before being integrated in the resulting module. The next definition gives the rewrite rules for flattening a whole ABS-VM program.

Definition 6.7 (Flattening an ABS-VM program). Let $\varepsilon$ denote the empty program, representing the initial partial result of flattening an ABS-VM program Prg. The rules define a judgment of the form Prg' $, A_{1}, D_{1} \stackrel{\text { Prg }}{\longrightarrow}$ Prg' ' $, A_{2}, D_{2}$, where: Prg' (either $\varepsilon$ or a variability-free ABS program) is a partial result of the flattening of Prg; the set $A_{1}$ identifies the already generated variability-free ABS modules; the set $D_{1}$ identifies the variability-free ABS modules that must be generated to fulfill the dependencies in Prg'; the variability-free ABS program Prg' ' is obtained by adding to Prg' the code of one of the variability-free ABS modules identified by $D_{1}$; the sets $A_{2}$ and $D_{2}$ are obtained by suitably updating $A_{1}$ and $D_{1}$, respectively. Let $\stackrel{\operatorname{Prg}}{\longrightarrow}$ be the transitive closure of $\stackrel{\mathrm{Prg}}{\longrightarrow}$. The flattening of ABS-VM program Prg is the variability-free ABS program Prg' such that $\varepsilon, \emptyset, \emptyset \stackrel{\text { Prg }}{\longrightarrow} \operatorname{Prg}^{\prime}, A, \emptyset$ holds.

$$
\begin{aligned}
& \frac{\text { mdllnit(Prg })=M \quad M \stackrel{\operatorname{Prg}, \perp}{\longrightarrow} D, M d 1 \quad A=\{(M, \perp)\}}{\varepsilon, \emptyset, \emptyset \stackrel{\operatorname{Prg}}{\longrightarrow} M d 1, A,(D \backslash A)} \\
& \begin{array}{rl}
\operatorname{Prg}^{\prime} \neq \varepsilon \quad(M, x c) \in D_{1} & M \stackrel{\operatorname{Prg}, x c}{\longrightarrow} D, \operatorname{Mdl} \quad A_{2}=A_{1} \cup\{(M, x c)\} \quad D_{2}=\left(D_{1} \cup D\right) \backslash A_{2}
\end{array}
\end{aligned}
$$

The sets $A$ and $D$ above refer to dependencies in the original program Prg. The first rule starts with the empty ABS program and dependency sets, adds the ABS module implementing the (unique part of the) main module of Prg and updates the dependency sets. The second rule extends Prg' by adding the ABS module required by one of the dangling dependency in $D_{1}$, and replaces the dependency sets $A_{1}$ and $D_{1}$ by their updated versions $A_{2}$ and $D_{2}$.

\section{PROPERTIES OF VARIABILITY MODULES}

The following definition and theorem show that one can automatically infer the maximal set of class/interface declarations that can soundly be annotated with unique.

Definition 7.1 (Function MaxUNIQUE). For every program Prg, for all VM $\mathrm{M}$ of Prg, let $S_{M}=\operatorname{CORE}(\operatorname{Prg}, M) \backslash \operatorname{BASE}(\mathrm{Prg}, \mathrm{M})$ and define $F_{\mathrm{M}}:\left(2^{S_{\mathrm{M}}}, \subseteq\right) \rightarrow\left(2^{S_{\mathrm{M}}}, \subseteq\right)$ to be the non-increasing monotone function such that: $F_{M}(X)$ is the subset of $X$ obtained by removing simultaneosly all classes/interfaces M.N such that the definition of $N$ in the core part of $M$ contains a with-free-dependency on a class/interface M.N' $\notin X$ or contains a with-open-dependency. Then MaxUNIQUE(Prg,M) is the set computed by iterating $F_{M}(X)$ on $S_{M}$ until a fixpoint is reached, i.e., the set $U=F_{M}^{n}\left(S_{M}\right)$ such that $U=F_{M}(U)$ for some $n \geq 0$.

Observe that MaxUNIQUE(Prg, M) is computed locally on the VM $M$ and always terminates (since $F_{M}$ is non-increasing monotone and $S_{M}$ is finite). Unfortunately, a program Prg' such that, for all VM $M$ of $\operatorname{Prg}^{\prime}$, UNIQUE $\left(\mathrm{Prg}^{\prime}, \mathrm{M}\right)=$ MaxUNIQUE $\left(\mathrm{Prg}^{\prime}, \mathrm{M}\right)$ may not adhere to the PEV, because of item (3) in Def. 5.3. However, by the following theorem, if such a Prg' does not adhere to the PEV, then any program obtained from Prg' by adding or removing unique annotations does not adhere to the PEV.

THEOREM 7.2 (MAXIMAL SET OF UNIQUE ANNOTATIONS ENFORCING THE PEV). For all programs Prg adhering to the PEV: (i) for all $V M$ of $\operatorname{Prg} \mathrm{M}, \mathrm{UNIQUE}(\mathrm{Prg}, \mathrm{M}) \subseteq$ MaxUNIQUE(Prg,M); (ii) the program Prg' obtained by adding unique annotations to Prg until, for all $V M \mathrm{M}$, UNIQUE $\left(\mathrm{Prg}^{\prime}, \mathrm{M}\right)=$ MaxUNIQUE $\left(\mathrm{Prg}^{\prime}, \mathrm{M}\right)$, adheres to $\mathrm{PEV}$.

Proof. By Def. 7.1 $F_{\mathrm{M}}$ is a set-theoretic inclusion-preserving map and the powerset $2^{S_{\mathrm{M}}}$ is a complete lattice. By the Knaster-Tarski theorem [31] there exist smallest and greatest fixpoints of $F_{\mathrm{M}}$. Moreover, the PEV (Def. 5.3.(2)) requires that UNIQUE(Prg, $M$ ) is a fixpoint of $F_{M}$. Now item (i) holds, because MaxUNIQUE(Prg, M) is the greatest fixpoint of $F_{\mathrm{M}}$-the proof is as follows: let $G$ be the greatest fixpoint of $F_{\mathrm{M}}$; clearly $G \subseteq S_{\mathrm{M}}$ and (since $F_{\mathrm{M}}$ is non-increasing monotone) $G=F_{M}^{n}(G) \subseteq F_{M}^{n}\left(S_{M}\right)$ for all $n \in \mathbb{N}$; but MaxUNIQUE(Prg, M) is the fixpoint obtained by iterating $F_{M}$ on $S_{M}$. Item (ii) holds, because Prg' satisfies Def. 5.3-in particular: item (1) holds by definition of $S_{\mathrm{M}}$ and non-increasing monotonicity of $F_{\mathrm{M}}$; item (2) is satisfied by any fixpoint of $F_{M}$; since Prg satisfies item (3), so does Prg'.

Definition 7.3 (Soundness of Delta-application). Let м be a VM of Prg. Then $\mathrm{m}$ is delta-application sound in Prg, if for all $x c \in\{\perp\} \cup\{\pi \mid$ $\pi$ is a product of $\mathrm{M}\}$ there exists a VM Core Mdic and a set $D$ such that $\mathrm{M} \stackrel{\operatorname{Prg}, x c}{\longrightarrow} D$, Mdic holds. Prg is delta-application sound, if all VMs $\mathrm{M}$ in Prg are delta-application sound.

Recall that programs are considered equal modulo permutation of class/interface definitions, field/method definitions, etc.

Theorem 7.4 (Flattening SoundNess). If program Prg in normal form adheres to the PEV and is delta-application sound, then $\varepsilon, \emptyset, \emptyset \stackrel{\text { Prg }}{\longrightarrow}$ $\mathrm{Prg}^{\prime}, A, \emptyset$ for some variability-free ABS program $\mathrm{Prg}$ '.

Proof. First we note that the rules in Def. 6.7 can be applied only a finite number of times, because the set of possible dependencies in Prg is finite (bounded by the set of variants per module). Thus termination is ensured.

The fact that Prg is in normal form guarantees: (i) all VM dependencies are defined in Prg; (ii) all configuration expressions KE in syntactic dependencies are valid products of the corresponding VM. These two facts ensure that all dependencies in Prg correspond to an actual dependency $(M, x c)$ where $M$ is declared in Prg and $x c$ is either $\perp$ or a product of $M$. In particular, if we consider any rewriting sequence $\varepsilon, \emptyset, \emptyset \stackrel{\text { Prg }}{\longrightarrow} \operatorname{Prg}^{\prime}, A, D$, all pairs $(M, x c)$ in $A, D$ are such that $x c$ is either $\perp$ or a product of $M$. 


\section{IMPLEMENTATION AND EVALUATION}

\subsection{Integration into the ABS Tool Chain}

We implemented the VM concept as part of the ABS compiler tool chain (with exception of open product definitions, currently under development). The implementation is available. ${ }^{3}$ The readme in the repository describes how to access the case studies.

To integrate VMs into the ABS compiler tool chain, only the frontend (parser and preprocessor) needed to be changed. This is, because flattening (Sect. 6.2) produces variability-free ABS code, keeping ABS code generation and semantic analysis (type checking) as is. The ABS parser's grammar is extended with the constructs described in Sect. 4. As expected, ABS's existing delta application mechanism (including calls to original (...)) could be fully reused. The implementation also includes: (i) the normal form check (Def. 4.1), with error reporting in case it is violated (not yet fully implemented); (ii) the PEV check (Sect. 5) with error reporting in case PEV is violated; (iii) the flattening mechanism described in Sect. 6.2; (iv) adjustment of the feature model (needed, because VMs use a simpler feature modeling language than ABS's $\mu T V L$ [6]).

\subsection{Case Studies}

ABS-VM was used in four case studies-the source code of the studies is available at the URL given above.

The first case study models from scratch a portal to compare insurance services [29]. It contains a product line model with three VMs in nearly 700 lines of code with eight features. ${ }^{4}$ It uses VMs to model different insurance offers. Their interoperability is required so that users can compare them in the portal.

The remaining three case studies either refactor or extend existing ABS models using the VM concept. Each of them illustrates a different use of VMs to support interoperable variants:

VMs vs. external tool chain. The AISCO system uses an external ad-hoc mechanism in Java to mimic variable modules in ABS. In Sec. 8.2.1 we compare the original system with our reimplementation with VMs.

VMs vs. exploiting traits. The FormbaR model relied on specific patterns to handle interoperability through the class model and traits. ${ }^{5}$ We show in Sec. 8.2.2 how the relevant parts of FormbaR are remodeled with VMs.

VMs vs. standard ABS SPLs. Using an ABS model of weak memory (Sec. 8.2.3), we show that without VMs, one would need to manually duplicate several modules.

8.2.1 AISCO. AISCO (Adaptive Information System for Charity Organizations) [40] is a modular web portal that supports the business processes (information, reporting, spending, expenditure) of charity organizations. It consists of an SPL implemented in ABS and its variability reflects the differing legal and operational requirements of the organizations. The code is in production at https://aisco.splelive.id/.

\footnotetext{
${ }^{3}$ https://zenodo.org/record/5045008

${ }^{4}$ The model contains four further features for the legacy SPL mechanism of ABS, kept for backwards compatibility.

${ }^{5}$ Traits $[16,34]$ are sets of methods that can be added to a class. The ABS-VM implementation supports traits. Since traits are orthogonal to the notion of VM we have not included them in the fragment of ABS-VM formalized in this paper. We refer to Damiani et al. [9] for a presentation of the notion of traits supported by ABS.
}

Evaluation. The requirements stipulate co-existence of multiple ABS variants, for example, different formats for financial reports. As this is not supported in current SPL approaches including ABS, an ad-hoc Java framework on top of ABS handled interoperability at runtime. For the case study, the main aspects of AISCO were re-implemented in ABS-VM in 160 lines of code, one VM with four features and five different deltas for financial reporting. All variants can interoperate within one and the same program generated from the ABS-VM code, instead of relying on an external, non-generic framework that is deeply interwined with the ABS model.

8.2.2 FormbaR. This is a re-modeling of the industrial FormbaR case study [24] (the basis of Sect. 2). VMs are useful to model infrastructure elements, such as signals coming in different variants that must coexist and interoperate within the same infrastructure model. The FormbaR model uses one class per infrastructure element, but this relies on the fact that in this case classes are a sufficient unit for variability. The domain is modelled as a tree-like type structure - additional constraints are imposed only implicitly. The part of the model that involves interoperable variability has been re-implemented using VMs.

Evaluation. The partial refactoring showed that by introducing a VM with five features (Main, Pre, Speed, Signal, Pov) and seven deltas, the total number of lines for the five remodeled kinds of signal ${ }^{6}$ is reduced from 241 to 180 (-25\%). Excluding the lines of code needed only for variability modeling (configuration knowledge and delta headers), the remodeled part has 163 lines (-33\%). The original model [24] uses traits to reduce code duplication in the implementation of the different kinds of signals. The ABS-VM reformulation of the model does not need to use traits. The reformulated model is: (i) shorter (in terms of length of code), because in the original model there is a separate class for each kind of signal; and (ii) more comprehensible: the feature model captures constraints in the model that were implicit before (for example, that two traits should not be used by the same class) and it declaratively connects code variability to the domain model.

8.2.3 Weak Memory Models. This case study is the VM extension of an ABS model of weak memory [23]. In sequentially consistent memory models all read- and write-accesses of some code are processed in the order they are issued. Weakly consistent (for short: weak) memory models allow partial or complete re-ordering of accesses to increase efficiency. The ABS weak memory model formalizes different relaxation strategies and device models, so as to allow to simulate and analyze their effects. An weak memory model in the case study is a class that manages a list of memory accesses. Variability includes different types of reordering (read before write, etc.). We extended the existing ABS model to two devices with two memory systems each: any of the four memory models can be different.

Evaluation. The ABS-VM model contains one module for memory models and one for devices (i.e., pairs of memory models). For comparison, we implemented this with product lines based on standard DOP. As we potentially need four different memory models, this required to copy the memory model module including all deltas four times. Furthermore, the device module had to be copied twice.

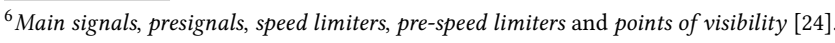


Essentially, we must perform manually part of the VM flattening until we can rely standard operations on the modules.

The ABS-VM version has $485 \mathrm{LoC}$, of which $440 \mathrm{LoC}$ are the two variable modules. The standard ABS version has $1322 \mathrm{LoC}$ $(+272 \%)$, of which $620 \mathrm{LoC}$ are concerned with deltas and variability and 582 are the core product of the modules for memory models and devices. We refrained from reducing code duplication through traits to illustrate that product line systems without native support of interoperable variants can only replicate this behavior through massive code duplication.

If a module has $p$ products, then in ABS-VM only $p$ configurations are declared, for any number of used variants. Another observation is that to connect $n$ variants, one needs to declare $p^{n}$ products: one for each combination. Hence, in addition to the additional delta declarations, this blows up the feature model unreasonably.

\section{RELATED WORK}

On Programming Constructs for MPLs and Variant Interoperability. Schröter et al. [36] advocate the use of suitable interfaces to support compositional analysis of MPLs, consisting of feature-oriented programming $(\mathrm{FOP})^{7}$ SPLs of Java programs, during different stages of the development process. Damiani et al. [14] informally outlined an extension of DOP to implement MPLs of Java programs by proposing linguistic constructs for defining an MPL as an SPL that imports other SPLs. In their proposal the feature model and artifact base of the importing SPL is entwined with the feature models and artifact bases of the imported SPLs. Therefore, in contrast to VMs, the proposal does not support encapsulation at SPL level. More recently, Damiani et al. $[12,13]$ formalized an extension of DOP to implement MPLs in terms of a core calculus, where products are written in an imperative version of Featherweight Java [19, 20]. The idea was to lift to the SPL level the use of dependent feature models to capture MPLs, as advocated by Schröter et al. [35, 37]. Like the earlier paper [14], the proposed SPL construct [13] models dependencies among different SPLs at the feature model level: to use two (or more) SPLs together, one must compose their feature models. In contrast, VMs do not require feature model composition.

The proposals mentioned above do not support variant interoperabilty [11]. Setyautami et al. [38] addressed variant interoperability at the level of of static UML class diagrams. In this paper we consider executable Java-like code.

Variant interoperability in terms of ABS code is addressed in our previous work [11], where we considered a set of product lines, each comprising a set of modules. However, in that proposal, encapsulation is not realized by mechanisms at the module level (as in VMs): unique declarations are supported (unsatisfactorily) by common modules (which is not fine-grained enough), and the concepts of modularity (through modules) and variability (through product lines) are interwined. In contrast, the VM concept proposed in this paper unifies modules and product lines by adding the capability to model variability directly to modules: each module is a product line, each product line is a module. This drastically simplifies the language, yet allows more far-reaching reuse of the DOP

${ }^{7}$ FOP [1, Sect. 6.1], [3], [25] can be characterized as the restriction of DOP, where there is a one-to-one mapping between deltas and features (each delta is activated if and only if the corresponding feature is selected), the application order is total, and there are no class/interface/field/method removal operations [33]. mechanism natively supported by ABS. Furthermore, VMs ease the cognitive burden of variability modeling, extending a common module framework, instead of adding another layer on top.

On Variability-aware Module Systems. Kästner et al. [26] propose a variability-aware module system (that we call VAMS in the following) for procedural languages. Like in our proposal, each VAMS module is an SPL. However, there are four important conceptual differences, which we outline in the following:

(i) VAMS does not encapsulate variability (cf. Sect. 5). Namely, modules import function declarations without specifying the modules from which they should be imported. In order to generate a variant, VAMS requires the user to write a composition expression, which lists all the modules to be composed and resolves dependencies and ambiguities (e.g., when a module imports a function that is defined in two different modules) by specifying how functions are renamed or hidden (and how features are renamed, selected or deselected). So, VAMS is not concerned with explicit dependencies between modules, which are crucial to usability and central to the PEV introduced in this work. By exploiting PEV, VMs achieve simplicity and usability: configuring a single $\mathrm{VM}$ м triggers automatic generation of all required variants of $\mathrm{m}$ and other VMs.

(ii) The design of VAMS does not target variant interoperability (Kästner et al. [26] do not mention this issue). Making two variants of the same module to co-exist, requires to create copy of the module and to rename (possibly by exploiting the module composition language provided by VAMS) all its features and all its exported functions. Instead, providing usable support to variant interoperability is a central design goal of VM.

(iii) VAMS variability is achieved explicitly by using an annotative approach: code elements (import/export declarations and function declarations) are annotated with presence conditions (propositional formulas over features). In VM variability is achieved explicitly by DOP for class/interface declarations and implicitly for export/import declarations.

(iv) VAMS is formalized by building on a calculus in the spirit of Cardelli's module system formalization [5] for procedural programming languages, where a module consists of a set of imported typed function declarations and a list of typed function definitions, and is implemented as a module system for $\mathrm{C}$ code. Therefore, VAMS is tailored for procedural language, where the interface of each module describes names and types of imported and exported functions, and there is a global function namespace. Moreover, even though each module has its own feature model, there is a global feature namespace. In contrast, VMs target Java-like languages, are based on the module system of ABS [17, 21] (a fairly standard module system close to Java and Haskell) and are implemented as an extension of the ABS module system. Each VM has a local namespace (which reduces overhead), also features are local to VMs.

On Variability Modules in Java. The paper [39] suggests that VM can be implemented on top of any Java-like language with modest effort. The solution ${ }^{8}$ presented there takes a different approach from the present account: it dispenses with explicit language constructs to model variability, but uses only standard Java constructs. This is

\footnotetext{
${ }^{8}$ The VM concept presented in [39] is based on the research reported here, but was published earlier due to the uncertainties inherent to peer-reviewed publication.
} 
achieved with an architectural pattern: delta application is achieved with decorators, the name space is managed with abstract factories, and each product is a module declaration in itself. The sole reliance on standard Java constructs comes with some limitations: unique class/interface declarations are not directly supported (but can be achieved by suitable final annotations). In consequence, the PEV is not enforced. Open product declarations are not supported. Unsoundness of a product might only be detected at runtime, because reflection is used for module name resolution instead of flattening. Therefore, [39] does not feature a formal semantics of VM and family-based checking.

\section{CONCLUSION AND FUTURE WORK}

This work presents variability modules, a novel approach to implement MPLs consisting of DOP SPLs of Java-like programs, where different, possibly interdependent, variants of the same SPL can coexist and interoperate. The PEV allows to encapsulate variability mechanisms by standard modules.

We are currently extending the implementation (cf. Sect. 8.1) to support: (i) open product definitions; and (ii) a family-based analysis $[15,42,43]$ that, given an ABS-VM program Prg, checkswithout actually generating any variant-whether all variants of the VMs in Prg can be generated and, as a whole, form a well-typed variability-free ABS program (implying that Prg can be flattened and is well-typed).

In future work we would like to validate the extended tool chain by further developing the use cases (cf. Sect. 8.2) and by considering novel use cases.

\section{A DELTA APPLICATION RULES}

In this appendix we present the rules describing the application of an ordered sequence of deltas $\overline{\mathrm{Dlt}}$ to to a sequence of interface/class definitions $\overline{\overline{D e f n}}($ see Definition 6.4).

The rules, given in Fig. 7, fall into the following categories:

Rules for a Sequence of Deltas describe how to apply each of the deltas in a sequence. D:EMPTY removes the delta if no operations are left to execute. Rules D:INTER and D:Class extracts the first interface/class operation from the delta and applies it to the list of definition. D:END concludes the application process when the sequence of the deltas to be applied is empty.

Rules for a Delta describe how to apply the actions specified by a delta to a whole class or interface definition. Rule D:ADDsI adds an interface by adding its definition to the list of definitions. Rule D:REMsI removes an interface by looking up its definition using the name from the delta modifier. The rules for classes, D:ADDsC and D:RemsC are analogous. Rules D:ModI and D:ModC modify an interface, or class, by applying the rules for interface modifiers (or class modifiers).

Rules for Extends/Implements Clauses modify the extends clauses of interfaces and implements clauses of classes. by removing (D:EM:REMs) or adding (D:EM:ADDs) it. Rule D:EM:EMPTY is applied if all modification of the clause have been applied.

Rules for Interfaces modify interfaces. Rule D:I:ЕMPTY is applicable when no further modification is requested on the given interface, so that the result is the interface itself. Rule D:I:ADDs adds the specified method header to the interface (provided that no header with this name is already present in the interface). Rule D:I:REMs removes an existing method header from the interface.

Rules for Classes modify classes. They are very similar to the ones for interfaces, with two exceptions: first, manipulation of method headers is replaced by manipulation of fields (rules D:C:ADDsF and D:C:REMSF) and methods implementations (rules D:C:ADDsM and D:C:RemsM). Second, methods may be modified using rule D:C:Mods. This rule replace the method implementatation, but keeps the old implementation with a fresh name. If the new implementation contains an original statement, then this statement is replaced by a call to the old implementation.

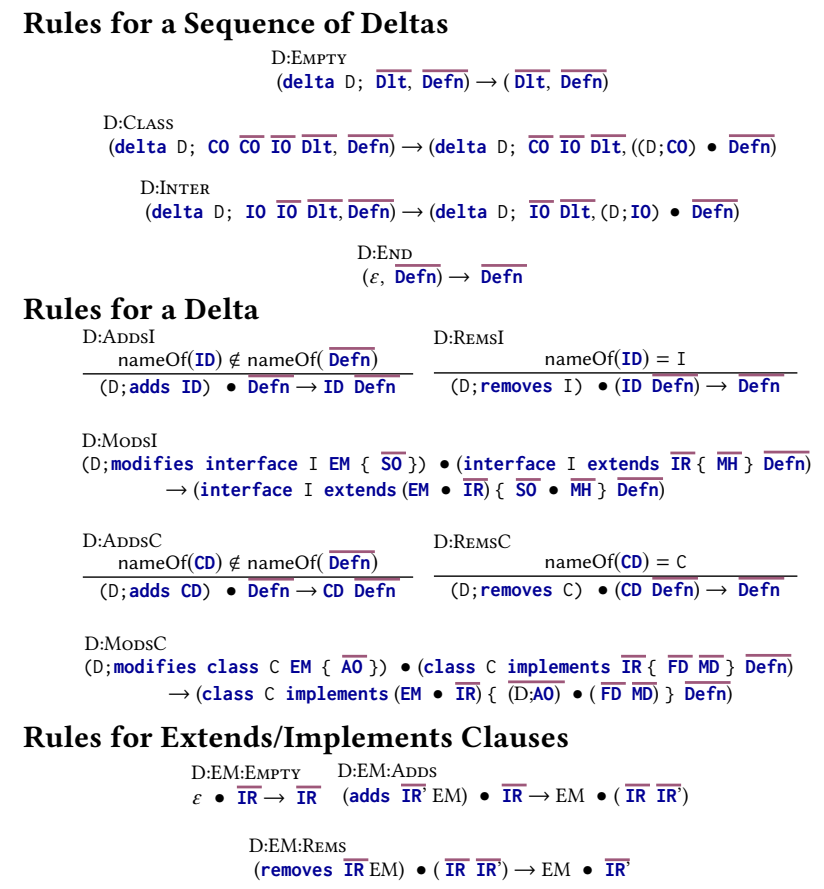

Rules for Interfaces
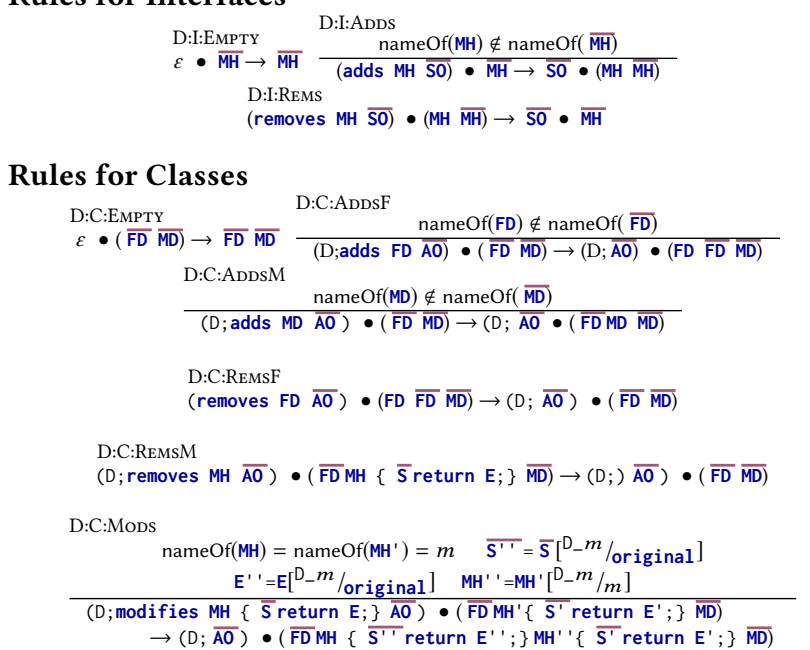

Figure 7: Delta Application Rules 


\section{REFERENCES}

[1] Sven Apel, Don S. Batory, Christian Kästner, and Gunter Saake. 2013. FeatureOriented Software Product Lines: Concepts and Implementation. Springer. I-XVI, 1-315 pages.

[2] Don Batory. 2005. Feature Models, Grammars, and Propositional Formulas. In Proceedings of International Software Product Line Conference (SPLC) (LNCS, Vol. 3714). Springer, 7-20. https://doi.org/10.1007/11554844_3

[3] Don Batory, Jacob Neal Sarvela, and Axel Rauschmayer. 2004. Scaling StepWise Refinement. IEEE Transactions on Software Engineering 30 (2004), 355-371. https://doi.org/10.1109/TSE.2004.23

[4] Lorenzo Bettini, Ferruccio Damiani, and Ina Schaefer. 2013. Compositional type checking of delta-oriented software product lines. Acta Informatica 50, 2 (2013), 77-122. https://doi.org/10.1007/s00236-012-0173-z

[5] Luca Cardelli. 1997. Program Fragments, Linking, and Modularization. In Proceedings of the 24th ACM SIGPLAN-SIGACT Symposium on Principles of Programming Languages (Paris, France) (POPL '97). ACM, New York, NY, USA, 266-277. https://doi.org/10.1145/263699.263735

[6] Dave Clarke, Radu Muschevici, José Proença, Ina Schaefer, and Rudolf Schlatte. 2010. Variability Modelling in the ABS Language. In FMCO (Lecture Notes in Computer Science, Vol. 6957). Springer, 204-224. https://doi.org/10.1007/978-3642-25271-6 11

[7] P. Clements and L. Northrop. 2001. Software Product Lines: Practices \& Patterns Addison Wesley Longman.

[8] Krzysztof Czarnecki and Ulrich W. Eisenecker. 2000. Generative Programming: Methods, Tools, and Applications. Addison-Wesley.

[9] Ferruccio Damiani, Reiner Hähnle, Eduard Kamburjan, and Michael Lienhardt 2017. A Unified and Formal Programming Model for Deltas and Traits. In FASE (Lecture Notes in Computer Science, Vol. 10202). Springer, 424-441. https://doi. org/10.1007/978-3-662-54494-5_25

[10] Ferruccio Damiani, Reiner Hähnle, Eduard Kamburjan, and Michael Lienhardt 2018. Interoperability of software product line variants. In SPLC. ACM, 264-268. https://doi.org/10.1145/3233027.3236401

[11] Ferruccio Damiani, Reiner Hähnle, Eduard Kamburjan, and Michael Lienhardt. 2018. Same Same But Different: Interoperability of Software Product Line Variants In Principled Software Development. Springer, 99-117. https://doi.org/10.1007/9783-319-98047-8_7

[12] Ferruccio Damiani, Michael Lienhardt, and Luca Paolini. 2017. A Formal Model for Multi SPLs. In FSEN (Lecture Notes in Computer Science, Vol. 10522). Springer, 67-83. https://doi.org/10.1007/978-3-319-68972-2 5

[13] Ferruccio Damiani, Michael Lienhardt, and Luca Paolini. 2019. A formal model for Multi Software Product Lines. Science of Computer Programming 172 (2019), 203 - 231. https://doi.org/10.1016/j.scico.2018.11.005

[14] Ferruccio Damiani, Ina Schaefer, and Tim Winkelmann. 2014. Delta-oriented Multi Software Product Lines. In Proceedings of the 18th International Software Product Line Conference - Volume 1 (Florence, Italy) (SPLC '14). ACM, 232-236. https://doi.org/10.1145/2648511.2648536

[15] Benjamin Delaware, William R. Cook, and Don Batory. 2009. Fitting the Pieces Together: A Machine-checked Model of Safe Composition. In ESEC/FSE (Amsterdam, The Netherlands). ACM, 243-252. https://doi.org/10.1145/1595696.1595733

[16] Stéphane Ducasse, Oscar Nierstrasz, Nathanael Schärli, Roel Wuyts, and Andrew P. Black. 2006. Traits: A mechanism for fine-grained reuse. ACM Trans. Program. Lang. Syst. 28, 2 (2006), 331-388. https://doi.org/10.1145/1119479.1119483

[17] Reiner Hähnle. 2013. The Abstract Behavioral Specification Language: A Tutoria Introduction. In Intl. School on Formal Models for Components and Objects: Post Proceedings (LNCS, Vol. 7866), Marcello Bonsangue, Frank de Boer, Elena Giachino, and Reiner Hähnle (Eds.). Springer, 1-37. https://doi.org/10.1007/978-3-64240615-7 1

[18] Gerald Holl, Paul Grünbacher, and Rick Rabiser. 2012. A systematic review and an expert survey on capabilities supporting multi product lines. Information and Software Technology 54, 8 (2012), 828 - 852. https://doi.org/10.1016/j.infsof.2012. 02.002

[19] A. Igarashi, B. Pierce, and P. Wadler. 2001. Featherweight Java: A Minimal Core Calculus for Java and GJ. ACM TOPLAS 23, 3 (2001), 396-450. https: //doi.org/10.1145/503502.503505

[20] Atsushi Igarashi, Benjamin C. Pierce, and Philip Wadler. 1999. Featherwieght Java: A Minimal Core Calculus for Java and GJ. In Proceedings of the 1999 ACM SIGPLAN Conference on Object-Oriented Programming Systems, Languages \& Applications (OOPSLA '99), Denver, Colorado, USA, November 1-5, 1999, Brent Hailpern, Linda M. Northrop, and A. Michael Berman (Eds.). ACM, 132-146. https://doi.org/10.1145/320384.320395

[21] Einar Broch Johnsen, Reiner Hähnle, Jan Schäfer, Rudolf Schlatte, and Martin Steffen. 2010. ABS: A Core Language for Abstract Behavioral Specification. In Formal Methods for Components and Objects - 9th International Symposium, FMCO 2010, Graz, Austria, November 29 - December 1, 2010. Revised Papers. 142-164. https://doi.org/10.1007/978-3-642-25271-6 8

[22] Eduard Kamburjan and Reiner Hähnle. 2016. Uniform Modeling of Railway Operations. In FTSCS (Communications in Computer and Information Science,
Vol. 694). 55-71. https://doi.org/10.1007/978-3-319-53946-1 4

[23] Eduard Kamburjan and Reiner Hähnle. 2018. Prototyping Formal System Models with Active Objects. In Proceedings 11th Interaction and Concurrency Experience, ICE 2018, Madrid, Spain, fune 20-21, 2018 (EPTCS, Vol. 279), Massimo Bartoletti and Sophia Knight (Eds.). 52-67. https://doi.org/10.4204/EPTCS.279.7

[24] Eduard Kamburjan, Reiner Hähnle, and Sebastian Schön. 2018. Formal Modeling and Analysis of Railway Operations with Active Objects. Science of Computer Programming 166 (Nov. 2018), 167-193. https://doi.org/10.1016/j.scico.2018.07.001

[25] Kyo Kang, Sholom Cohen, James Hess, William Novak, and A. Peterson. 1990. Feature-Oriented Domain Analysis (FODA) Feasibility Study. Technical Report CMU/SEI-90-TR-021. Software Engineering Institute, Carnegie Mellon University, Pittsburgh, PA. http://resources.sei.cmu.edu/library/asset-view.cfm?AssetID= 11231

[26] Christian Kästner, Klaus Ostermann, and Sebastian Erdweg. 2012. A Variabilityaware Module System. In Proc. ACM International Conference on Object Oriented Programming Systems Languages and Applications (Tucson, Arizona, USA) (OOPSLA '12), Gary T. Leavens and Matthew B. Dwyer (Eds.). ACM, New York, NY, USA, 773-792. https://doi.org/10.1145/2384616.2384673

[27] Michael Lienhardt and Dave Clarke. 2012. Conflict Detection in Delta-Oriented Programming. In ISoLA 2012, Heraklion, Crete, Greece, October 15-18, 2012, Proceedings, Part I (Lecture Notes in Computer Science, Vol. 7609). Springer, 178-192. https://doi.org/10.1007/978-3-642-34026-0_14

[28] Reza Mauliadi, Maya R. A Setyautami, Iis Afriyanti, and Ade Azurat. 2017. A platform for charities system generation with SPL approach. In Proc. Intl. Conf. on Information Technology Systems and Innovation (ICITSI) (Bandung, Indonesia). IEEE, New York, NY, USA, 108-113. https://doi.org/10.1109/ICITSI.2017.8267927

[29] Melissa Mendoza. 2020. Variability-aware Modules. Master's Thesis.

[30] K. Pohl, G. Böckle, and F. van der Linden. 2005. Software Product Line Engineering - Foundations, Principles, and Techniques. Springer, Berlin, Germany.

[31] S. Roman. 2008. Lattices and Ordered Sets. Springer New York. https://books. google.it/books?id=NZN8aum26LgC

[32] Ina Schaefer, Lorenzo Bettini, Viviana Bono, Ferruccio Damiani, and Nico Tanzarella. 2010. Delta-Oriented Programming of Software Product Lines. In Software Product Lines: Going Beyond (SPLC 2010) (LNCS, Vol. 6287). 77-91. https://doi.org/10.1007/978-3-642-15579-6_6

[33] Ina Schaefer and Ferruccio Damiani. 2010. Pure delta-oriented programming. In Proceedings of the 2nd International Workshop on Feature-Oriented Software Development (Eindhoven, The Netherlands) (FOSD '10). ACM, 49-56. https: //doi.org/10.1145/1868688.1868696

[34] Nathanael Schärli, Stéphane Ducasse, Oscar Nierstrasz, and Andrew P. Black. 2003. Traits: Composable Units of Behaviour. In ECOOP 2003 - Object-Oriented Programming, 17th European Conference, Darmstadt, Germany, July 21-25, 2003, Proceedings (Lecture Notes in Computer Science, Vol. 2743), Luca Cardelli (Ed.). Springer, 248-274. https://doi.org/10.1007/978-3-540-45070-2 12

[35] Reimar Schröter, Sebastian Krieter, Thomas Thüm, Fabian Benduhn, and Gunter Saake. 2016. Feature-model Interfaces: The Highway to Compositional Analyses of Highly-configurable Systems. In Proceedings of the 38th International Conference on Software Engineering (Austin, Texas) (ICSE '16). ACM, 667-678. https://doi. org $/ 10.1145 / 2884781.2884823$

[36] Reimar Schröter, Norbert Siegmund, and Thomas Thüm. 2013. Towards Modular Analysis of Multi Product Lines. In Proceedings of the 17th International Software Product Line Conference Co-located Workshops (Tokyo, Japan) (SPLC'13). ACM, 96-99. https://doi.org/10.1145/2499777.2500719

[37] Reimar Schröter, Thomas Thüm, Norbert Siegmund, and Gunter Saake. 2013. Automated analysis of dependent feature models. In The Seventh International Workshop on Variability Modelling of Software-intensive Systems, VaMoS '13, Pisa, Italy, Fanuary 23 - 25, 2013. 9:1-9:5. https://doi.org/10.1145/2430502.2430515

[38] Maya R. A Setyautami, Daya Adianto, and Ade Azurat. 2018. Modeling Multi Software Product Lines Using UML. In Proc. 22nd Intl. Systems and Software Product Line Conference, vol. 1 (Gothenburg, Sweden). ACM, New York, NY, USA, 274-278. https://doi.org/10.1145/3233027.3236400

[39] Maya Retno Ayu Setyautami and Reiner Hähnle. 2021. An Architectural Pattern to Realize Multi Software Product Lines in Java. In Proc. 15th Intl. Working Conf. on Variability Modelling of Software-Intensive Systems, Krems, Austria, Paul Grünbacher, Christoph Seidl, Deepak Dhungana, and Helena Lovasz-Bukvova (Eds.). ACM Press, 9:1-9:9. https://doi.org/10.1145/3442391.3442401

[40] Maya R. A. Setyautami, Rafiano R. Rubiantoro, and Ade Azurat. 2019. ModelDriven Engineering for Delta-Oriented Software Product Lines. In 26th AsiaPacific Software Engineering Conf., APSEC, Putrajaya, Malaysia. IEEE, 371-377. https://doi.org/10.1109/APSEC48747.2019.00057

[41] Chico Sundermann, Michael Nieke, Paul Maximilian Bittner, Tobias Heß, Thomas Thüm, and Ina Schaefer. 2021. Applications of \#SAT Solvers on Feature Models. In VaMoS'21: 15th International Working Conference on Variability Modelling of Software-Intensive Systems, Virtual Event / Krems, Austria, February 9-11, 2021. 12:1-12:10. https://doi.org/10.1145/3442391.3442404

[42] Sahil Thaker, Don Batory, David Kitchin, and William Cook. 2007. Safe Composition of Product Lines. In Proceedings of the 6th International Conference on Generative Programming and Component Engineering (Salzburg, Austria) (GPCE 
'07). ACM, New York, NY, USA, 95-104. https://doi.org/10.1145/1289971.1289989

[43] Thomas Thüm, Sven Apel, Christian Kästner, Ina Schaefer, and Gunter Saake 2014. A Classification and Survey of Analysis Strategies for Software Product Lines. ACM Comput. Surv. 47, 1, Article 6 (2014), 45 pages. https://doi.org/10. $1145 / 2580950$

[44] Guadalupe Isaura Trujillo-Tzanahua, Ulises Juárez-Martínez, Alberto Alfonso Aguilar-Lasserre, and María Karen Cortés-Verdín. 2018. Multiple Software Product Lines: applications and challenges. In Trends and Applications in Software Engineering, Jezreel Mejia, Mirna Muñoz, Álvaro Rocha, Yadira Quiñonez, and Jose Calvo-Manzano (Eds.). Springer International Publishing, Cham, 117-126. https://doi.org/10.1007/978-3-319-69341-5 11

[45] Niklaus Wirth. 1980. The module: A system structuring facility in high-level programming languages. In Language Design and Programming Methodology, Jeffrey M. Tobias (Ed.). Springer Berlin Heidelberg, Berlin, Heidelberg, 1-24. https://doi.org/10.1007/3-540-09745-7_1

[46] Peter Y. H. Wong, Nikolay Diakov, and Ina Schaefer. 2012. Modelling Distributed Adaptable Object Oriented Systems using HATS Approach: A Fredhopper Case Study (invited paper). In 2nd International Conference on Formal Verification of Object-Oriented Software, Torino, Italy (LNCS, Vol. 7421). Springer. https: //doi.org/10.1007/978-3-642-31762-0_5 